\title{
NHANES Data indicates that adequate vitamin intake remains a challenge for a large part of the elderly even in affluent societies
}

\author{
Barbara Troesch $^{1}$, Michael McBurney ${ }^{2}$, Peter Weber $^{1}$, Manfred Eggersdorfer ${ }^{*}$
}

${ }^{1}$ DSM Nutritional Products Ltd, Kaiseraugst, Switzerland

${ }^{2}$ DSM Nutritional Products Ltd, Parsippany, NJ, United States

*Corresponding author: Manfred Eggersdorfer, DSM Nutritional Products Ld. Wurmisweg 576, 4303 Kaiseraugst, Switzerland,Tel: +41 61815 8196; Fax: +41 61815 8490; E-mail: manfred.eggersdorfer@dsm.com

\begin{abstract}
Background and aims: Demographic changes lead to an increased number of elderly, which has a dramatic impact on health care cost. One factor driving up this cost is the widespread malnutrition in elderly, especially in patients, already before entering the health care system. The aim of this paper was to analyze the adequacy of vitamin intakes in older people based on data from the US National Health and Nutrition Examination Survey (NHANES) 2003 to 2008.

Methods: Vitamin intake for the US elderly aged $>70$ years was determined based on information collected during NHANES 2003-2008. The proportions of elderly with intakes below the Estimated Average Requirement (EAR) and the correlation with household incomes were calculated for each vitamin.

Results: $>50 \%$ US elderly do not reach the EAR for vitamin D, E and K and $35-40 \%$ for vitamin $\mathrm{C}$ and $\mathrm{A}$, while for the B-vitamins, the proportion ranges from $1-30 \%$ and vitamin intakes correlated with household incomes.

Conclusions: Even in an affluent country such as the US, a high number of elderly do not get enough vitamins from their diets. Affordable solutions to supply them with necessary vitamins are needed to counter possible adverse effects on health and wellbeing.
\end{abstract}

Received Date:August 19, 2015

Accepted Date: January 20, 2016

Published Date: January 26, 2016

Citation: Eggersdorfer, M., et al. Adequate Vitamin Intake Remains a Challenge for a Large Part of the Elderly Even In Affluent Societies. (2016) Int J Food Nutr Sci 3(1): 189-194.

Keywords: Aging/elderly; Vitamins; Deficiencies; Vitamin intake; Nutritional inadequacies; Dietary surveys

DOI: $10.15436 / 2377-0619.16 .035$

Abbreviations: AI: Adequate Intake; CDC: Center for Disease Control and Prevention; EAR: Estimated Average Requirement; NCHS: National Center for Health Statistics; NCI: National Cancer Institute; NHANES: National Health and Nutrition Examination Survey; USDA: United States Department of Agriculture

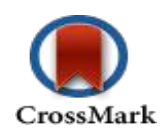

\section{Introduction}

In aging societies health care cost puts an increasing burden on national budgets and finding ways to reduce them are high on the list of governments' priorities. One area that is getting increasing attention is the cost of malnutrition in health care settings as well as nursing homes for the elderly and infirm. It was estimated that the annual cost of managing patients at risk of disease-related malnutrition in the United Kingdom in 2003 was $£ 7.3$ billion $^{[1]}$. If these figures are extrapolated to Europe, the cost increases to $€ 170$ billion per year ${ }^{[2]}$. Already in 2003, two thirds of this money was spent on the care of individuals aged $>65$ years ${ }^{[1]}$. This age group is expected to increase from 506 million or 7\% of the world's population in 2008 to 1.3 billion or 14\% in $2040^{[3]}$, which will have an enormous impact on the cost linked to their care.

A recent survey in hospitals showed that while malnutrition as defined by the Malnutrition Universal Screening Tool was generally high in all persons, the older ones had a $40 \%$ higher risk compared to the ones younger than 65 years ${ }^{[4]}$. Moreover, it is thought that $25 \%$ of patients admitted to hospitals have a poor nutritional status at arrival ${ }^{[2]}$. A recent study in the USA found that malnourished elderly or older people at risk of malnutrition were more likely to need health care services and to visit hospitals 
and emergency rooms ${ }^{[5]}$. Consequently, the risk of malnutrition should already be addressed before the person enters into the care system due to disease, disability or injury.

So far, the focus of malnutrition screening assessment has largely been on protein-energy malnutrition. However, inadequate vitamin intakes have recently been reported to be widespread in Western countries in the population aged 20 to 50 years of age ${ }^{[6]}$. Therefore, our aim was to assess the vitamin intakes of population aged $>70$ years surveyed during the United States National Health and Nutrition Examination Survey (NHANES) for 2003 to 2008 to gain insight into the adequacy of vitamin intake in the elderly population before they enter the care system. The NHANES data is to our knowledge the only such data set for the age group of $>70$ years available to such an extent to the public. However, the conclusions drawn from this analysis may be relevant for other affluent Western countries, even though differences exist between the nutritional habits and food intakes in the US and Europe as well as between different European countries.

\section{Methods}

\section{Study population}

We focused and used the dataset from NHANES 2003-2008 for people aged $>70$ years as reference. This survey uses a complex, multistage probability sampling design to obtain a sample representative of the civilian non-institutionalized household population of the United States ${ }^{[7-9]}$. In this part of the survey data are selected for all adult participants with complete and reliable dietary records in the age ranges $>70$ years $(n=$ 2'545). The NHANES data are collected by the National Center for Health Statistics (NCHS) of the Centers for Disease Control and Prevention (CDC). All participants or proxies provided written informed consent, and the Research Ethics Review Board at the NCHS approved the survey protocol. Information on dietary intakes were collected during an in-person examination using a 24-hour recall, while demographic data, such as age, gender and household income were collected through a computer-assisted personal interview. A second 24-h dietary recall was collected via telephone around 3 to 10 days later. The measures used to estimate portion sizes are described elsewhere ${ }^{[10]}$. To be able to assess the quality of the diet alone, the vitamin intakes from dietary sources and fortification excluding dietary supplements were used.

\section{Nutrients from foods}

The NHANES total diet files report combined intakes of vitamins in food consumed by NHANES participants as analyzed by CDC/ US Department of Agriculture (USDA) using various USDA databases (The Food and Nutrient Database for Dietary Studies was used for NHANES 2003-2004 (version 2.0), 2005-2006 (version 3.0), and 2007-2008 (version 4.0)) to determine the vitamin content of NHANES foods.

\section{Analyses of NHANES 2003-2008 Dataset}

Intake data from both $24-\mathrm{h}$ recalls were used to estimate usual intake and percentiles of intake from foods using the $\mathrm{Na}$ tional Cancer Institute $(\mathrm{NCI})$ method $^{[11]}$. The balanced repeated replication approach was implemented in conjunction with NCI method to develop standard errors and confidence intervals. Co- variates in the usual intake models included day of the week of dietary recall (weekend/weekday) and interview sequence of the dietary recall (in person vs. via telephone). The population was stratified by gender and was grouped by age for all NCI runs.

\section{Adequacy of vitamin intake}

US Dietary Reference Intakes distinguish between two levels of intakes: The Estimated Average Requirement (EAR), which is defined as the intake that covers the needs of $50 \%$ of the respective age and gender group and the Recommended Daily Allowance, which are thought to cover the needs of $97.5 \%$ of that population group ${ }^{[12-15]}$. If insufficient evidence is available to arrive at such values (E.g. for vitamin K), Adequate Intakes (AI) are defined instead ${ }^{[12]}$. The EAR (or AI in the case of vitamin $\mathrm{K}$ ) for each vitamin (Table 1$)^{[12-15]}$ were used to quantify the potential gap in vitamin intakes in the US elderly according to the cut-point method that defines intakes below the EAR as inadequate ${ }^{[16]}$. As the revised recommendations for vitamin $D$ had not been published when the data was analyzed, but it was clear that the review was underway, it was decided not to use the previous recommendations ${ }^{[17]}$. Instead, the adequacy of intake was established by comparing the new $\mathrm{RDA}^{[14]}$ with the percentiles of vitamin $\mathrm{D}$ intake in this population.

Table 1: Estimated Average Requirements (EAR) for men ( $n=1$ '274) and women $(n=1$ '271) in the United States for the age group of $>70$ years ${ }^{[12-15]}$ and proportion of male and female seniors ( $>70$ years) in the NHANES cohort 2003 to 2008 with vitamin intakes below the age-specific EAR

\begin{tabular}{|l|c|c|c|c|c|}
\hline & & \multicolumn{2}{|c|}{ EAR } & \multicolumn{2}{c|}{$<$ EAR } \\
\hline Vitamin & Unit/d & Men & Women & Men & Women \\
\hline Vitamin A & $\mu \mathrm{g} \mathrm{RE}$ & 625 & 500 & $69.9 \%$ & $66.8 \%$ \\
\hline Vitamin D & $\mu \mathrm{g}$ & 10 & 10 & $>90 . \%^{1}$ & $>90 . \%^{1}$ \\
\hline Vitamin $\mathrm{E}$ & $\mathrm{mg} \mathrm{TE}$ & 12 & 12 & $99.1 \%$ & $99.7 \%$ \\
\hline Thiamine & $\mathrm{mg}$ & 1.0 & 0.9 & $14.4 \%$ & $30.3 \%$ \\
\hline Riboflavin & $\mathrm{mg}$ & 1.1 & 0.9 & $5.6 \%$ & $9.8 \%$ \\
\hline Niacin & $\mathrm{mg}$ & 12 & 12 & $11.3 \%$ & $22.4 \%$ \\
\hline Vitamin $\mathrm{B}_{6}$ & $\mathrm{mg}$ & 1.4 & 1.3 & $31.2 \%$ & $48.3 \%$ \\
\hline Vitamin $\mathrm{B}_{12}$ & $\mu \mathrm{g}$ & 2.0 & 2.0 & $2.4 \%$ & $11.0 \%$ \\
\hline Folic acid & $\mu \mathrm{g}$ & 320 & 320 & $19.5 \%$ & $41.7 \%$ \\
\hline Vitamin $\mathrm{C}^{1}$ & $\mathrm{mg}$ & 75 & 60 & $50.6 \%$ & $47.1 \%$ \\
\hline Vitamin $\mathrm{K}^{*}$ & $\mu \mathrm{g}$ & 120 & 90 & $82.2 \%{ }^{3}$ & $66.0 \%{ }^{3}$ \\
\hline
\end{tabular}

RE, retinol equivalent; TE, tocopherol equivalent. the $90^{\text {th }}$ percentile of Vitamin D intakes were found to be clearly below the revised EAR for this vitamin, ${ }^{2}$ The Institute of Medicine concluded that insufficient evidence was available to arrive at an EAR for vitamin K and therefore only defined an Adequate Intake

\section{Impact of income on vitamin intake}

The data was stratified by household income to assess the impact of available budget on vitamin intakes. The proportion with vitamin intakes below the EAR were then compared between individuals with yearly household incomes $<\$ 25^{\prime} 000$, $\$ 25^{\prime} 000$ to $\$ 75^{\prime} 000$ and $>\$ 75^{\prime} 000$. For this, the Cochran-Armitage trend test, a modified Chi-squared test for ordered categorical data, was used ${ }^{[18]}$. $\mathrm{P}$ values $<0.05$ were considered significant. 


\section{Results}

The sample contained 1'274 men and 1'271 women aged $>70$ whose data on dietary intake was collected during 2003 to 2008 . Table 1 shows the proportion of elderly men and women in the US with vitamin intakes below the EAR. While some small differences between the genders are evident, the results show that more than half of the people do not reach the EAR for vitamin D, E and $\mathrm{K}$ and $35 \%$ to $40 \%$ for vitamin $C$ and $\mathrm{A}$. The situation is a bit better for the B-vitamins, with levels of inadequate intake varying from $\sim 1 \%$ for vitamin $\mathrm{B} 12$ in men to $\sim 30 \%$ for vitamin B6 in women. There is a significant trend towards reduced intakes of vitamins with lower household income in the elderly in the US (Table 2).

Table 2: Proportion of population with vitamin intakes below the Estimated Average Requirement (EAR) by household budget

\begin{tabular}{|c|c|c|c|c|c|c|c|c|}
\hline & \multicolumn{2}{|c|}{$\begin{array}{l}\text { Low } \\
\text { income }^{1}\end{array}$} & \multicolumn{2}{|c|}{$\begin{array}{l}\text { Medium } \\
\text { income }^{2}\end{array}$} & \multicolumn{2}{|c|}{$\begin{array}{l}\text { High } \\
\text { income }\end{array}$} & \multirow[t]{2}{*}{$\begin{array}{l}\text { P- } \\
\text { value }\end{array}$} & \multirow[t]{2}{*}{$\begin{array}{l}\Delta \\
{[\%]^{4}}\end{array}$} \\
\hline & $\begin{array}{l}\% \\
<\text { EAR }\end{array}$ & $\mathrm{SE}$ & $\begin{array}{l}\% \\
<\text { EAR }\end{array}$ & $\mathrm{SE}$ & $\begin{array}{l}\% \\
<\text { EAR }\end{array}$ & $\mathrm{SE}$ & & \\
\hline Vitamin A & 40.5 & 1.9 & 34.1 & 2.0 & 30.0 & 5.2 & $<0.001$ & 25.9 \\
\hline Thiamine & 12.7 & 1.4 & 9.1 & 1.1 & 3.8 & 1.6 & $<0.001$ & 70.1 \\
\hline Riboflavin & 4.2 & 0.5 & 2.7 & 0.4 & 1.4 & 0.7 & $<0.001$ & 66.7 \\
\hline Niacin & 7.9 & 1.4 & 4.7 & 0.8 & 1.0 & 0.7 & $<0.001$ & 87.3 \\
\hline Vitamin B6 & 31.1 & 2.2 & 23.1 & 1.9 & 13.7 & 3.2 & $<0.001$ & 55.9 \\
\hline Folic Acid & 19.0 & 1.7 & 12.7 & 1.3 & 9.5 & 2.6 & $<0.001$ & 50.0 \\
\hline Vitamin $B_{12}$ & 5.6 & 1.3 & 2.2 & 0.6 & 2.4 & 1.5 & $<0.001$ & 59.3 \\
\hline Vitamin C & 39.1 & 2.2 & 33.1 & 1.9 & 22.9 & 3.3 & $<0.001$ & 41.4 \\
\hline Vitamin $^{5}$ & na & na & na & na & na & na & na & na \\
\hline Vitamin E & 98.4 & 0.5 & 96.3 & 0.8 & 91.8 & 2.5 & $<0.001$ & 6.7 \\
\hline Vitamin $\mathrm{K}^{6}$ & 75.3 & 1.7 & 71.6 & 2.2 & 56.7 & 8.0 & $<0.001$ & 24.7 \\
\hline
\end{tabular}

${ }^{1}$ Household income $<\$ 25^{\prime} 000 /$ year, $n=1151,{ }^{2}$ Household income $\$ 25^{\prime} 000$ to $\$ 75^{\prime} 000$ per year, $n=1011,{ }^{3}$ Household income $>\$ 75^{\prime} 000$ per year, $n=204,{ }^{4}$ Relative difference in the proportion of persons below recommendations in the highest and the lowest income group, ${ }^{5} \mathrm{This}$ value could not be calculated as the Dietary Reference Values for vitamin D were under revision when the data was analysed, ${ }^{6}$ The Institute of Medicine concluded that insufficient evidence was available to arrive at an EAR for vitamin $\mathrm{K}$ and therefore only defined an Adequate Intakena: not available

\section{Discussion}

The information collected in the dietary intake survey as part of NHANES 2003-2008 indicates that the majority of elderly people in the United States do not met the EAR for at least one vitamin: $>50 \%$ US elderly do not reach the EAR for vitamin D, E and $\mathrm{K}$ and 35 to $40 \%$ for vitamin $\mathrm{C}$ and $\mathrm{A}$, while for the B-vitamins, the proportion ranges from 1 to $30 \%$ (Table 1). The results from the European Nutrition and Health Report indicate that the situation of the elderly in the general population is similar in Europe ${ }^{[19]}$ : Average intakes of vitamin D and folate for the elderly in most of the 15 countries included in the report were found to be below recommendations, while vitamin $\mathrm{E}$ and $\mathrm{C}$ were below recommendations in around half of them ${ }^{[19]}$. In an Austrian cohort of non-institutionalized persons aged 70 to 90 years, inadequate plasma levels were found in a significant proportion of the cohort for various vitamins ${ }^{[20]}$. A multi-centre study in various European countries identified vitamin D and B12 as particularly critical in their elderly cohort ${ }^{[21]}$. It is also in agreement with a survey that classified around $50 \%$ of community dwelling elderly in Europe as at risk for malnutrition ${ }^{[22]}$.

Intake are clearly improved by the use of nutritional supplements: The risk to have vitamin intakes below the EAR was four times lower in elderly persons who regularly used supplements of one or more micronutrients ${ }^{[23]}$. In elderly men, the prevalence of vitamin A, E and folate intakes below the EAR decreased from $53 \%$ to $4 \%$, from $93 \%$ to $14 \%$ and from $75 \%$ to $7 \%$, respectively with the use of supplements ${ }^{[23]}$. However, only around $3 \%$ of elderly persons in German nursing homes were taking vitamin supplements ${ }^{[24]}$. In comparison, more than $35 \%$ of people aged 60 or more in the US were reported to be taking them ${ }^{[25]}$. A similar situation probably exists for micronutrients supplied by fortification and enrichment, as these provide a significant source for vitamins and minerals in the $\mathrm{US}^{[26]}$, but not in Europe $^{[27]}$.

The NHANES survey did not collect information on institutionalized elderly; however, data collected in Germany shows that the situation of elderly in German institutions is worse in such a cohort: Vitamin intakes for more than $80 \%$ of institutionalized elderly in Germany are inadequate for vitamin C, D, E, and folic acid as well as for thiamine in women ${ }^{[24]}$. With the exception of niacin in men, the intakes for all the other vitamins assessed in this study were found to be inadequate for $25 \%$ to $75 \%$. While distinct differences can be expected between the nutritional situation in Germany and the US, comparison with the non-institutionalized German seniors ${ }^{[28]}$ shows that the situation is clearly worse for those in care settings ${ }^{[24]}$. Even though the controlled environment of a hospital or care home would be ideal for improving nutrition, this opportunity has so far been not been taken due to lack of awareness of the problem not just in the general public, but also among health care professionals and decision makers ${ }^{[2]}$. Consequently, it has been postulated that nutrition took a more prominent role in the training of physicians and other health care professionals ${ }^{[29]}$.

Old age and the accompanying pathologies result in a multitude of physiological and social changes. Older people tend to eat alone more often, which was shown to result in decreased food intakes ${ }^{[30]}$. Due to physical disabilities, dementia, depression or other psychological factors, they are less capable of preparing meals for themselves ${ }^{[31]}$. A recent study showed that disability and chronic illness significantly increased the odds of a diet characterised by low variability ${ }^{[32]}$. Moreover, decreased effectiveness to detect and react to hunger further increases the risk of malnutrition ${ }^{[30]}$. Appetite tends to decrease due to declining taste and smell sensitivities, various pathological conditions or medications and impaired chewing due to ill-fitting dentures $^{[33]}$. Reduced secretion of saliva in the elderly or the intake of certain drugs makes swallowing more difficult ${ }^{[34]}$.

However, inadequate vitamin status is often not only caused by decreased intakes, but also impaired absorption. As an example, the intakes of vitamin B12 were reported to be mostly above the EAR (Table 1) even without intakes from supplements. However, data collected in NHANES 1999 to 2004 showed that 5 to $25 \%$ of people aged 60 or more had low serum vitamin B12 levels ${ }^{[35]}$. In Germany, were intakes are lower still, vitamin B12 deficiency can be expected to be even more common. It is thought that around two thirds of cases of cobalamin 
deficiency in the elderly are due to malabsorption ${ }^{[36,37]}$. Given the relative size of cobalamin stores, a large time lag exists between the onset of the malabsorption and the development of the deficiency ${ }^{[38]}$. Even subclinical deficiency is an indicator of insufficient absorption of dietary cobalamin and oral supplementation with daily doses of at least $1000 \mu \mathrm{g}$ was proposed for elderly people at risk of malabsorption ${ }^{[39,40]}$.

Changes in body composition as part of the aging process are associated with an increased risk for malnutrition as they lead to decreased energy requirements ${ }^{[41]}$ while requirements for micronutrients remain the same ${ }^{[12,13,15]}$ or, for vitamin $\mathrm{D}$, increase ${ }^{[14]}$. A study in healthy elderly found intakes below EAR for most people for vitamin D and calcium and for around half for vitamin B6, folate, magnesium and zinc, while energy intake corresponded with the recommendations ${ }^{[42]}$. This highlights the importance of nutrients dense foods for population groups with low levels of activity and therefore reduced energy intakes $^{[43]}$. However, a study comparing recommendations with actual eating behaviour showed that among the $>70$ years, recommended intakes for fruits, vegetables and whole grains were not meet by $\sim 70 \%,>80 \%$ and $>90 \%$ of individuals, respective$1 y^{[44]}$. It has also been shown that nutrient density decreased in parallel with the household budget available for food ${ }^{[45]}$. Given the often reduced budgets of the elderly, this adds a further difficulty to achieving a balanced diet with sufficient micronutrients. This relationship between household budget and vitamin intake was confirmed in our data (Table 2).

The relevance of these vitamin inadequacies for life quality and health care cost has been studied in most detail for the case of vitamin $\mathrm{D}^{[46-50]}$. Among the patients in a medical intensive care unit, vitamin D deficiency at admission was as high as $78 \%$ and 25 -hydroxy vitamin $\mathrm{D}$ levels were found to correlate with mortality after $\geq 2$ days in the hospital ${ }^{[51-54]}$. Vitamin D status was inversely related to hospital stay and 25-hydroxy vitamin D levels were found to decrease further during the stay at the surgical intensive care unit ${ }^{[55]}$. As a consequence of the increased time spent at the hospital, treatment cost more than doubled for people with severe vitamin $\mathrm{D}$ deficiency ${ }^{[55]}$.

Data from such large-scale dietary intake surveys are considered to provide the most accurate data on populations currently available, as validated biomarkers with generally accepted cut-off points only exist for a few vitamins. Comparison between countries has its limitations, as, although the concept of intake recommendations is widely recognized, national dietary reference values vary considerably in terminology and recommended level ${ }^{[56-59]}$. Moreover, day-to-day variance in nutrient intake typically results in large within-individual variability when conducting dietary surveys using methods such as the 24-hour recall. However, some of this bias can be eliminated by using the NCI method to estimating usual intakes based on data from at least two interviews per person ${ }^{[60]}$. The method has been shown to provide a good approximation of usual intake, especially given to relatively large sample size ${ }^{[61]}$. To assess adequacy of intake from such data, the Institute of Medicine recommends to use the proportion of a population with intakes below the EAR, as individual requirements are normally not known ${ }^{[16,62]}$. When different cut-offs (RDA, 0.66 RDA, 0.50 RDA and EAR) were compared with more comprehensive analyses, defining inadequacy of intake as intakes lower than the EAR gave the most accurate estimates ${ }^{[63]}$. Still, one limitation of the paper is that dietary surveys do not provide a direct measure of an individual's nutrient status, but such data is still missing for large-scale cohorts.

\section{Conclusion}

The data presented shows that intakes are critically low in the elderly in the US for various vitamins. Given the importance of adequate vitamin intakes and the problems achieving them from the diet alone, specific fortified foods and supplements targeted at the elderly, both healthy and frail, need to be developed and tested. It has been shown that the addition of an oral nutrient supplement containing vitamins and additional energy to the diet of acutely ill elderly patients led to an increase in nutritional status ${ }^{[64]}$ and quality of life ${ }^{[65]}$ combined with a decrease in hospital re-admission ${ }^{[66]}$ and depressive symptoms ${ }^{[67]}$. Given the complexity of the physiological and metabolic changes inherent to the aging process, more research is warranted to gain a better understanding of the effects of vitamin inadequacies on aging and the capacity of nutritional interventions to slow this process.

\section{Competing Interests}

The authors are employed by DSM Nutritional Products Ltd., a bulk supplier of vitamins.

\section{Authors' Contributions}

BT, PW and ME defined the scope of the paper; BT analysed the data; BT and PW wrote the paper; BT, PW and ME had primary responsibility for the final content; all authors read and approved the final manuscript.

\section{Acknowledgment}

We would like to thank Victor Fulgoni III of Nutrition Impact, LLC for the statistical analysis of the NHANES 2003 to 2008 data and RotrautSchoop from DSM Nutritional Products Ltd. for her support with the analysis of the correlation between vitamin intakes and household budgets.

\section{References}

1. Russell, C.A. The impact of malnutrition on healthcare costs and economic considerations for the use of oral nutritional supplements. (2007) Clinical Nutrition Supplements 2(1): 25-32.

2. Ljungqvist, O., de Man, F. Under nutrition: A major health problem in europe. (2009) Nutri Hosp 24(3): 369-370.

3. Kinsella, K., He, W. An aging world: 2008 International population reports. (2009) National Institute of Health, National Institute of Aging. 4. Russell, C., Elia, M. Nutrition screening survey in the uk in 2008. (2009) Nutrition Screening Week Survey and Audit.

5. Yang, Y., Brown, C.J., Burgio, K.L., et al.Undernutrition at baseline and health services utilization and mortality over a 1-year period in older adults receiving medicare home health services. (2011) J Am Med Dir Assoc 12(4): 287-294.

6. Troesch, B., Hoeft, B., McBurney, M., et al. Dietary surveys indicate vitamin intakes below recommendations are common in representative western countries. (2012) Brit J Nutr 108(4): 692-698.

7. Nhanes 2003 - 2004. Centers for Disease Control and Prevention, National Center for Health Statistics.

8. Nhanes 2005 - 2006. Centers for Disease Control and Prevention, National Center for Health Statistics.

9. Nhanes 2007 - 2008. Centers for Disease Control and Prevention, 
National Center for Health Statistics.

10. Millum, J., Wendler, D., Emanuel, E.J. The $50^{\text {th }}$ anniversary of the declaration of helsinki: Progress but many remaining challenges. (2013) JAMA 310(20): 2143-2144.

11. Tooze, J.A., Kipnis, V., Buckman, D.W., et al. A mixed-effects model approach for estimating the distribution of usual intake of nutrients: The nci method. (2010) Stat Med 29(27): 2857-2868.

12. Dietary reference intakes of vitamin a, vitamin k, arsenic, boron, chromium, copper, iodine, iron, manganese, molybdenum, nickel, silicon, vanadium, and zinc. (2001) National Academic Press.

13. Dietary reference intakes of vitamin c, vitamin e, selenium, and carotenoids. (2000) National Academic Press.

14. Dietary reference intakes for calcium and vitamin D. (2011) National Academies Press.

15. Dietary reference intakes for thiamin, riboflavin, niacin, vitamin $B_{6}$, folate, vitamin $B_{12}$, pantothenic acid, biotin and cholin. (1998) National Academic Press.

16. Dietary reference intakes: Applications in dietary planning. (2003) National Academy Press.

17. Institute of Medicine: Dietary reference intakes for calcium, phosphorus, magnesium, vitamin d and fluoride. (1997) National Academic Press.

18. Agresti, A. An introduction to categorical data analysis $2^{\text {nd }} e d n$. (2007) John Wiley and Sons.

19. Elmadfa, I. Meyer, A., Nowak, V., et al. European nutrition and health report 2009. (2009) Karger.

20. Fabian, E., Bogner, M., Kickinger, A., et al. Vitamin status in elderly people in relation to the use of nutritional supplements. (2011) J Nutr Health Aging 16(3): 206-212.

21. De Groot, C.P., Van Staveren, W.A.Undernutrition in the europeanseneca studies. (2002) Clin Geriatr Med 18(4): 699-708.

22. De Morais, C.,. Oliveira, B., Afonso, C., et al. Nutritional risk of european elderly. (2013) Eur J Clin Nutr 67(11): 1215-1219.

23. Sebastian, R.S., Cleveland, L.E., Goldman, J.D., et al. Older adults who use vitamin/mineral supplements differ from nonusers in nutrient intake adequacy and dietary attitudes. (2007) J Am Diet Assoc 107(8): 1322-1332.

24. Diet of older people in inpatient facilities (serious - studies). (2008) Nutrition Report 2008 Bonn 157-204.

25. Rock, C.L. Multivitamin-multimineral supplements: Who uses them? (2007) Am J Clin Nutr 85(1): 277S-279S.

26. Fulgoni, V.L., Keast, D.R., Bailey, R.L., et al. Foods, fortificants, and supplements: Where do americans get their nutrients? (2011) J Nutr 141(10): 1847-1854.

27. Flynn, A., Hirvonen, T., Mensink, G.B., et al. Intake of selected nutrients from foods, from fortification and from supplements in various european countries. (2009) Food Nutr Res 53: 1-51.

28. Nationale Verzehrs Studie II. (2008) Max Rubner-Institut.

29. Lenders, C.M., Deen, D.D., Bistrian, B., et al. Residency and specialties training in nutrition: A call for action. (2014) The American Journal of Clinical Nutrition 99(4).

30. De Castro, J.M., Stroebele, N. Food intake in the real world: Implications for nutrition and aging. Clin Geriatr Med 18(4): 685-697.

31. Johnson, K.A., Bernard, M.A., Funderburg, K. Vitamin nutrition in older adults. (2002) Clin Geriatr Med 18(4): 773-799.

32. Labadarios, D., Steyn, N.P., Nel, J. How diverse is the diet of adult south africans? (2011) Nutr J10:33.

33. Russell, R.M., Rasmussen, H. The impact of nutritional needs of older adults on recommended food intakes. (1999) Nutrition in Clinical Care 2(3): 164-176.

34. D’Souza, A.L. Ageing and the gut. (2007) Postgrad Med J 83(975): 44-53.

35. Bailey, R.L., Carmel, R., Green, R., et al. Monitoring of vitamin $\mathrm{b}-12$ nutritional status in the united states by using plasma methylmalonic acid and serum vitamin b-12. (2011) Am J Clin Nutr 94(2): 552-561. 36. Carmel, R. Current concepts in cobalamin deficiency. (2000) Annu Rev Med 51: 357-375.
37. Andrès, E., Loukili, N.H., Noel, E., et al. Vitamin b12 (cobalamin) deficiency in elderly patients. (2004) Can Med Assoc J 171(3): 251259.

38. Carmel, R. Nutritional anemias and the elderly. (2008) Semin Hemato 145(4): 225-234.

39. Carmel, R. Efficacy and safety of fortification and supplementation with vitamin b12: Biochemical and physiological effects. (2008) Food Nutr Bull 29(2 Suppl): S177-S187.

40. Lane, L., Rojas-Fernandez, C. Treatment of vitamin b(12)-deficiency anemia: Oral versus parenteral therapy. (2002) The Annals of Pharmacotherapy 36: 1268-1272.

41. Buffa, R., Floris, G.U., Putzu, P.F., et al. Body composition variations in ageing. (2011) Coll Antropol 35(1): 259-265.

42. Foote, J.A., Giuliano, A.R., Harris, R.B. Older adults need guidance to meet nutritional recommendations. (2000) J Am Coll Nutr 19(5): 628-640.

43. Blumberg, J. Nutritional needs of seniors. (1997) J Am Coll Nutr 16(6): 517-523.

44. Krebs-Smith, S.M., Guenther, P.M., Subar, A.F., et al. Americans do not meet federal dietary recommendations. (2010) J Nutr 140(10): 1832-1838.

45. Darmon, N., Ferguson, E.L., Briend, A. A cost constraint alone has adverse effects on food selection and nutrient density: An analysis of human diets by linear programming. (2002) J Nutr 132(12): 3764-3771. 46. Annweiler, C., Llewellyn, D.J., Beauchet, O. Low serum vitamin $\mathrm{d}$ concentrations in alzheimer's disease: A systematic review and meta-analysis. (2013) J Alzheimers Dis 33(3): 659-674.

47. Annweiler, C., Rolland, Y., Schott, A.M., et al. Serum vitamin d deficiency as a predictor of incident non-alzheimer dementias: A 7-year longitudinal study. (2011) Dement Geriatr Cogn Disord 32(4): 273-278. 48. Annweiler, C., Rolland, Y., Schott, A.M., et al. Higher vitamin d dietary intake is associated with lower risk of alzheimer's disease: A 7-year follow-up. (2012) J Gerontol A Biol Sci Med Sci 67: 1205-1211. 49. Buell, J.S., Dawson-Hughes, B. Vitamin d and neurocognitive dysfunction: Preventing "D"ecline? (2008) Mol Aspects Med 29(6): 415422.

50. Smith, A.D. The worldwide challenge of the dementias: A role for $b$ vitamins and homocysteine? (2008) Food Nutr Bull 29(2 Suppl): S143172.

51. Smith, A.D., Smith, S.M., de Jager, C.A., et al. Homocysteine-lowering by $b$ vitamins slows the rate of accelerated brain atrophy in mild cognitive impairment: A randomized controlled trial. (2010)PLos One 5(9): e12244.

52. Rinaldi, P., Polidori, M.C., Metastasio, A., et al. Plasma antioxidants are similarly depleted in mild cognitive impairment and in alzheimer's disease. (2003) Neurobiol Aging 24(7): 915-919.

53. Harris, E., Kirk, J., Rowsell, R., et al. The effect of multivitamin supplementation on mood and stress in healthy older men. (2011) Hum Psychopharmacol 26(8): 560-567.

54. Venkatram, S., Chilimuri, S., Adrish, M., et al. Vitamin D deficiency is associated with mortality in the medical intensive care unit. (2011) Crit Care 15(6): R292.

55. Matthews, L.R., Ahmed, Y., Wilson, K.L., et al. Worsening severity of vitamin d deficiency is associated with increased length of stay, surgical intensive care unit cost, and mortality rate in surgical intensive care unit patients. (2012) Am J Surg 204(1): 37-43.

56. Doets, E.L., de Wit, L.S., Dhonukshe-Rutten, R.A., et al. Current micronutrient recommendations in europe: Towards understanding their differences and similarities. Eur J Nutr 47(Suppl1): 17-40.

57. Pavlovic, M., Prentice, A., Thorsdottir, I., et al. Challenges in harmonizing energy and nutrient recommendations in europe. (2007) Ann Nutr Metab 51(2): 108-114.

58. Roman Vinas, B., Ribas Barba, L., Ngo, J., et al. Projected prevalence of inadequate nutrient intakes in europe. (2011) Ann NutrMetab 59(2-4): 84-95.

59. Matthys, C., van'tVeer, P., de Groot, L., et al.Eurrecas approach for estimating micronutrient requirements. (2011) Int J Vitam Nutr Res 


\section{1(4): 256-263.}

60. Tooze, J.A., Kipnis, V., Buckman, D.W., et al. A mixed-effects model approach for estimating the distribution of usual intake of nutrients: The nci method. (2010) Stat Med 29(27): 2857-2868.

61. Souverein, O.W., Dekkers, A.L., Geelen, A., et al.Comparing four methods to estimate usual intake distributions. (2011) Eur J Clin Nutr 65(Suppl 1): S92-S101.

62. Carriquiry, A.L. Assessing the prevalence of nutrient inadequacy. ( 1999) Public Health Nutr 2(1): 23-34.

63. De Lauzon, B., Volatier, J., Martin, A. A montecarlo simulation to validate the ear cut-point method for assessing the prevalence of nutrient inadequacy at the population level. (2004) Public Health Nutr 7(7): 893-900.
64. Gariballa, S., Forster, S., Walters, S., et al. A randomized, double-blind, placebo-controlled trial of nutritional supplementation during acute illness. (2006) The American Journal of Medicine 119: 693-699. 65. Gariballa, S., Forster, S. Dietary supplementation and quality of life of older patients: A randomized, double-blind, placebo-controlled trial. (2007) J Am Geriatr Soc 55(12): 2030-2034.

66. Gariballa, S.E., Forster, S.J., Powers, H.J. Effects of mixed dietary supplements on total plasma homocysteine concentrations (thcy): A randomized, double-blind, placebo-controlled trial. (2012) Int J VitamNutr Res 82(4): 260-266.

67. Gariballa, S., Forster, S. Effects of dietary supplements on depressive symptoms in older patients: A randomised double-blind placebo-controlled trial. (2007) Clin Nutr 26(5): 545-551. 